\title{
LAS COMPETENCIAS PROFESIONALES DEL MAESTRO DE PRIMARIA DESDE LA PERSPECTIVA DEL TUTOR DEL CENTRO DE PRÁCTICAS
}

\section{PROFESSIONAL COMPETENCIES OF THE PRIMARY TEACHER: A STUDY FROM THE PERSPECTIVE OF THE TUTOR OF PRACTICUM}

\author{
$M^{a}$ Luisa Barceló Cerdá \\ Centro Universitario Villanueva. Universidad Complutense de Madrid \\ Marta Ruiz-Corbella \\ Universidad Nacional de Educación a Distancia - UNED
}

\section{RESUMEN}

Una asignatura clave en la formación inicial de los maestros es el Prácticum, en el que los tutores de los centros de prácticas cumplen un papel esencial como nexo entre la universidad y la escuela. Desde este rol este estudio pretende conocer cuáles son las competencias profesionales del maestro de Educación Primaria desde la perspectiva de estos tutores y su percepción sobre el logro de las mismas en la formación que imparte este Grado. Con una muestra de 131 tutores, pertenecientes a centros educativos de diversa titularidad de la Comunidad de Madrid, se desarrolla un estudio descriptivo y transversal mediante cuestionario ad hoc, compuesto por cuarenta ítems y validado por expertos. Los hallazgos confirman la percepción favorable de los tutores respecto a la importancia de las competencias profesionales incluidas en la formación inicial de esta titulación, destacando, a su vez, las carencias formativas especialmente en la dimensión social y personal de su función profesional. Estos resultados, contrarios a las directrices supranacionales del maestro del siglo XXI y a las investigaciones que se están desarrollando, demandan una revisión del perfil del maestro acorde con la escuela del siglo XXI. Esta realidad apunta a que todavía existe una separación radical entre la formación inicial del maestro y las demandas de la sociedad. Analizar estas propuestas y redefinir los contenidos competenciales que deben estar presentes en el diseño del título del maestro de Primaria es una necesidad imperante por la que aboga este trabajo.

Palabras clave: competencias profesionales, maestro, tutor de Prácticum, 
Educación Primaria.

\section{ABSTRACT}

The Practicum is a crucial element in the initial education of teachers, where tutors play an essential role as a nexus between university and school. From that perspective, the current study intends to find out what the Primary-Schoolteacher professional competencies are, from the tutors' perspective and their perception of the achievement of those competencies in initial training. The research has been conducted with a sample of 131 tutors, from a range of private and public schools in the Community of Madrid. It has been a descriptive and transversal study, in which an ad hoc questionnaire - consisting of 40 items and validated by experts - has been used. The findings reveal that there is a favorable perception on the part of tutors as regards the importance of teaching competencies included in the initial education of university students of this degree. At the same time, the results highlight the formative deficiencies, especially at the social and personal dimension of the professional function. Those results —which differ from the 21 st century teacher's supranational guidelines and from the research that currently is being conducted-demand a revision of the teacher's profile, in accordance with the 21 st century school. This reality points to the idea that there is a gap between first teacher formation and the demands of society. The analysis of these proposals and the redefinition of the content of competencies, which have to be present in the design of the degree of Primary School teachers, is a prevailing necessity advocated by this study.

Key words: Teacher education, Practicum supervision, Primary School, Professional competencies.

\section{EL PRÁCTICUM UN ESPACIO PRIVILEGIADO DE APRENDIZAJE}

El modelo universitario asumido por el Espacio Europeo de Educación Superior (EEES) ha planteado el diseño y desarrollo de los planes de estudios desde el enfoque de la formación por y en competencias. Con esta orientación, los planes de formación inicial del profesorado de Educación Primaria se dirigen hacía la adquisición de aquellas que habilitan para el acceso y el ejercicio de la profesión docente (Rodríguez, Álvarez, Gil y Romero, 201 1; Ruiz-Corbella y Naval, 2012; Valle y Manso, 2013). Esta perspectiva de la formación inicial ha traído consigo un cambio metodológico y un nuevo enfoque didáctico para acometer los desafíos de este entramado formativo, a la vez que trata de responder a las exigentes demandas del contexto en el que 
debemos enseñar. Como indican Mendoza y Covarrubias (2014) "parece evidente que las múltiples transformaciones de la sociedad han provocado nuevos desafíos en la profesión docente, desafíos que se traducen -a su vez- en nuevos roles y atribuciones para el profesorado. Conocer estas funciones es trascendental no sólo para definir el perfil profesional de los docentes, sino también para determinar los distintos tipos de conocimientos indispensables para el desarrollo de sus competencias profesionales" (p. 3)

Un primer paso para abordar el contenido de esta formación estriba en determinar qué entendemos por competencia, ya que de esta se derivan las decisiones para el diseño y el desarrollo de cada título. Aunque las competencias profesionales han sido ampliamente desarrolladas en la literatura científica no se constata un consenso respecto a la definición y clasificación de las mismas, quizá debido a que el término tenga su origen en el mundo laboral y esté relacionado con el desempeño, lo que exigirá un cambio en el modo de afrontar la formación de nuestros estudiantes al percibir que únicamente poniendo en práctica cada competencia, es como se llega a ser competente en un contexto y área profesional determinado (Cano 2005; Freire, Teijeiro y Blázquez, 2012; Medina, 2009; Perrenoud, 2004; Tejada 2013; Zabalza, 2003). Como bien indica Tejada (2012) "en los procesos de formación basada en competencias, los procesos de aprendizaje que se favorecen deben orientarse hacia la acción del participante tomando como referente el marco organizativo en el que la situación de trabajo es situación de aprendizaje" (p. 20).

En consecuencia, se constata que toda competencia, desde su naturaleza pedagógica, debe caracterizarse por ser holística, integradora, dinámica y reflexiva. Lo que implica necesariamente una formación práctica incardinada en el diseño de cada título.

Sin ánimo de ser exhaustivos, a partir de los diferentes enfoques en torno al concepto de competencia (Cano, 2005, 2008; Lasnier, 2000; LevyLeboyer, 2003; Perrenoud, 2004; Sarramona, 2007; Tejada, 2009; Tobón, 2012; Zabalza, 2003), se identifican ciertas coincidencias que ayudan a perfilar los rasgos esenciales para configurar este constructo. Destaca su carácter teórico-práctico, pues cobran sentido cuando se ponen en funcionamiento combinando conocimientos, aptitudes, habilidades, actitudes y rasgos de personalidad ante tareas complejas. Además presentan siempre un enfoque integrador, que supone saber coordinar y combinar de forma pertinente objetivos, conocimientos, destrezas, valores, responsabilidad sobre los resultados y asunción de riesgos. La competencia además se caracteriza por ser definible en la acción. Es un saber hacer flexible y contextualizado, indisociable de la práctica que, 
además, ha de ser transferible y aplicable a situaciones y actividades distintas, lo que lleva implícito la reflexión. También se caracteriza por ser dinámica, ya que indica el potencial que tiene una persona para el desempeño. Su adquisición se inicia en la formación inicial desde un enfoque transversal que supera la exclusividad y parcelación a cualquier disciplina y continúan desarrollándose, necesariamente, en la práctica profesional.

Si se entiende que la enseñanza es una profesión, se justifica el estudio en torno al análisis de las competencias profesionales que deben lograr los futuros maestros en su formación inicial. Pero la profesionalidad no se agota en la formación inicial, ya que como afirma Perrenoud, el maestro "no tiene un conocimiento previo de la solución a los problemas que emergerán de su práctica habitual y cada vez que aparece uno tiene que elaborar esta solución sobre la marcha [...] pero todo ello sería imposible sin un saber amplio, saber académico, saber especializado y saber experto" (Perrenoud, 2004, p. 183). La profesionalidad del maestro, por tanto, exige ser y estar preparado para adquirir las competencias que tienen durante su formación inicial que, desde nuestro punto de vista, deben alcanzar todas las dimensiones de la persona, pues tan importante es el desarrollo intelectual y reflexivo como el técnico, el social y el ético. En definitiva, todos los elementos se tienen que articular para hablar de una persona competente, capaz de tomar decisiones en un contexto determinado, de reflexionar sobre su propia práctica, de realizar un trabajo de calidad y de transferilo a otras situaciones.

A partir de la revisión realizada, en esta investigación se considera que un maestro es competente cuando en el contexto de trabajo y en diferentes situaciones de la práctica diaria, sabe movilizar de un modo integrado conocimientos, habilidades y actitudes, con las cualidades personales necesarias para ejercer de un modo efectivo las funciones que exige la profesión docente. Y además es capaz de identificar, interpretar, argumentar y atender desde la reflexión los problemas que se le presentan en su trabajo profesional, colaborando con el centro educativo con responsabilidad y compromiso ético.

Si la profesionalidad tiene mucho que ver con la práctica, es evidente la relevancia de las prácticas externas en el desarrollo formativo de nuestros estudiantes. En efecto, esta materia es un elemento clave en la formación del maestro, tal y como han demostrado diversas investigaciones (González y Fuentes, 2011 ; Latorre y Blanco, 2011 ; Lawson, Çakmak, Gündüz, y Busher, 2015; Rodicio e Iglesias, 201 1; Tejada y Ruiz, 2013). Todas estas aportaciones han venido a confirmar el evidente potencial de este periodo para integrar la formación disciplinar universitaria con el sentido profesional del quehacer del profesor. Como 
indican Raposo y Zabalza, el Prácticum viene a ser una "situación de aprendizaje, una experiencia personal y profesional al mismo tiempo que es un componente curricular más, una parte sustantiva y obligada en gran parte de las titulaciones en la educación superior" (2011, p. 17). Esta experiencia práctica que llevan a cabo los futuros maestros durante su formación inicial es esencial para el desarrollo competencial del profesorado, encontrando en el Prácticum un espacio privilegiado de aprendizaje integral de la profesión y, por ende, de las competencias profesionales específicas de esta titulación al poder trabajar con profesionales de la educación en escenarios de trabajo reales (Zabalza, 2003).

En este contexto, la figura del tutor de prácticas ha de tener una especial relevancia en el engranaje del sentido formativo de esta materia, al ser quien canaliza y articula, junto con los equipos docentes, los aprendizajes de este espacio profesional en integración con el dominio disciplinar, pedagógico y didáctico estimulando la práctica inicial reflexiva que permita que cada estudiante perciba una formación adaptada al futuro escenario profesional (Rodicio e Iglesias, 2011). En definitiva, tal y como sostienen Melgarejo, Pantoja, y Latorre (2014), si existen unos buenos profesionales, tanto en la universidad como en los centros educativos donde los estudiantes realizan sus prácticas, se tiene mucho ganado. Lo importante es encontrar los espacios y las temáticas donde se pueda converger con un mismo lenguaje escuela y universidad, y en actuación coordinada, dando prioridad a las necesidades que demanda la sociedad actual. Escenarios "donde el estudiante tiene oportunidad de experimentar los aprendizajes, conocimientos y relaciones interpersonales, adquiridos en su formación (...) imbuidos de hábitos de reflexión, de comprensión de las incertidumbres existentes en el proceso del desempeño profesional o la actividad docente cotidiana" (Tejada y Carvalho, 2013, p. 1585). De ahí que sea relevante conocer la percepción de los docentes en ejercicio, tutores de estas prácticas en los centros de trabajo, sobre cuáles son las competencias profesionales del maestro de Educación Primaria, contrastando esta propuesta con su percepción sobre la formación que estamos dando en nuestras universidades y su nivel de logro en nuestros estudiantes.

De acuerdo con esta propuesta, este trabajo pretende alcanzar dos objetivos:

$\checkmark$ Analizar las competencias profesionales propuestas en los planes de estudio de Magisterio de Primaria y el nivel de importancia que les conceden los tutores del Prácticum. 
Conocer el nivel de logro de las competencias profesionales por parte de los estudiantes desde la percepción de los tutores del Prácticum.

Cabe acentuar que el interés y la actualidad del presente estudio puede ser confirmado por la existencia de distintas investigaciones que, desde diferentes perspectivas, analizan las competencias del perfil de la profesión docente (Ferrández y Sánchez 2014; García y Martín, 2011; González, Pesquero, Sánchez, Fernández, De Juanas, y Martín Del Pozo, 2010; Martín del Pozo, Fernández, González, y De Juanas, 2013; Pesquero, Sánchez, González, Martín, Guardia, Cervelló, Fernández, Martínez, y Varela, 2008; Valdemoros y Lucas 2014, entre otros). Si bien, en ninguna de estas se analiza desde la perspectiva del tutor en el centro de prácticas, es un actor clave en la formación inicial de este profesional de la educación. A la vez que debe actuar en estrecha colaboración con los demás agentes implicados en este escenario, unidos por "la preocupación por formar profesionales con capacidad para enfrentarse y dar respuesta profesional a problemas de la profesión" (Marcelo, 2011. p. 23).

\section{MÉTODO}

Esta investigación se apoya en un diseño no experimental con una metodología descriptiva. Para la recogida y análisis de datos se han empleado dos técnicas. En primer lugar, el análisis de contenido dirigido a la identificación de las competencias profesionales del maestro de Primaria a partir de los documentos más relevantes sobre esta temática. En segundo lugar, un cuestionario ad hoc que recoge la percepción de los profesores tutores en los centros de prácticas sobre la formación inicial adquirida por nuestros estudiantes de Magisterio.

\subsection{Participantes}

De una población de 160 maestros, tutores del Prácticum, de la Comunidad Autónoma de Madrid, se ha recogido la respuesta de 131 procedentes de centros públicos, privados y concertados de esta Comunidad. Todos ellos tutelaron las prácticas del segundo cuatrimestre del curso 2013-14 a los estudiantes de Grado de Educación Primaria. La muestra se caracteriza por estar representada mayoritariamente por centros concertados y privados (Tabla 1), con un porcentaje de experiencia docente heterogénea, si bien más del $60 \%$ de los participantes tienen más de 11 años de experiencia (Tabla 2). En cuanto al sexo, la distribución representa la proporcionalidad de esta profesión 46 hombres $(35,11 \%)$, frente a 85 mujeres $(64,88 \%)$. 
Tabla 1: Distribución de la muestra según la titularidad del centro

\begin{tabular}{cccc}
\hline \multicolumn{4}{c}{ Titularidad } \\
\hline $\mathrm{N}$ & Público & Privado & Concertado \\
\hline 131 & $17(13 \%)$ & $49(37,4 \%)$ & $65(49,6 \%)$ \\
\hline
\end{tabular}

Tabla 2: Distribución de la muestra según los años de experiencia docente

\begin{tabular}{ccccc}
\hline$<5$ & 5 y 10 años & 11 y 15 años & 6 y 20 años & $>20$ \\
\hline 13 & 40 & 24 & 16 & 38 \\
$(9,9 \%)$ & $(30,5 \%)$ & $(18,3 \%)$ & $(12,2 \%)$ & $(29 \%)$ \\
\hline
\end{tabular}

\subsection{Instrumento}

La recogida de datos se ha realizado con un cuestionario elaborado ad hoc a partir de los indicadores obtenidos de la revisión de los modelos teóricos de referencia (ANECA, 2004; Cano, 2007; Freire, Teijeiro y Blázquez, 2012; Perrenoud, 2010; Tejada, 2009). Estas competencias se han agrupado de acuerdo a cuatro dimensiones: la competencia cognitiva- saber-, la competencia didácticometodológica -saber hacer-, la competencia social -saber estar y convivir-y la personal-saber ser-. Todas ellas están interrelacionadas e integradas entre sí, a la vez que son necesarias para el desempeño profesional como maestro. Para categorizarlas se ha tenido en cuenta la propuesta de Delors (1996), el modelo de Bunk (1994) y la adaptación realizada por Echevarría $(2002,2005)$ (Figura 1).

En orden a estas cuatro dimensiones se elaboró el cuestionario, dividido en dos partes. La primera se refiere a variables de identificación socioprofesional de estos tutores (sexo, edad, titularidad del centro educativo, experiencia docente). La segunda está conformada por cuarenta ítems estructurados en las cuatro dimensiones mencionadas de diez ítems cada una, valorados por dos escalas de cuatro opciones ( $1=$ Nada; $2=$ Poco; $3=$ Suficiente; y $4=$ Mucho). Se ha estimado la conveniencia de emplear cuatro valores para comprometer a los participantes a que se pronuncien y no escojan la respuesta central siguiendo las orientaciones de Hernández, Fernández, y Baptista (2010). Una de estas escalas mide el grado de importancia de las competencias profesionales y la otra la percepción del nivel de logro de las mismas en función de la formación inicial recibida por los futuros maestros. 
Figura 1: Categorización de las competencias profesionales del Maestro de Educación Primaria. Tomado de Delors (1996), Bunk (1994) y Echevarría $(2002,2005)$.

\begin{tabular}{|c|c|c|c|}
\hline & Competencia cognitiva & $\begin{array}{c}\text { Competencia } \\
\text { Didáctico-metodológica }\end{array}$ & \\
\hline $\begin{array}{l}\bar{\Phi} \\
\text { ○ } \\
\dot{0}\end{array}$ & $\begin{array}{l}\text { - Domino de la disciplina que enseña } \\
\text { - Conocimiento del centro educativo } \\
\text { - Identificación con el centro educativo } \\
\text {-Conocimiento sobre la legislación educativa } \\
\text {-Creatividad } \\
\text {-Capacidad investigadora } \\
\text {-Capacidad crítica, autocrítica y reflexiva } \\
\text {-Capacidad de análisis y síntesis } \\
\text {-Conocimiento de los distintos modelos de } \\
\text { instrucción } \\
\text {-Conocimiento de la Psicología del Desarrollo }\end{array}$ & $\begin{array}{l}\text {-Capacidad pedagógica } \\
\text {-Orientación y Acción Tutorial } \\
\text {-Planificación y organización } \\
\text {-Conocimiento de estrategias de } \\
\text { aprendizaje } \\
\text {-Promoción de la Convivencia } \\
\text {-Evaluación del proceso de enseñanza- } \\
\text { aprendizaje } \\
\text {-Dominio de las TIC } \\
\text {-Conocimiento de distintas técnicas } \\
\text {-Atención a la Diversidad } \\
\text {-Orientación académica y personal el } \\
\text { alumnado }\end{array}$ & 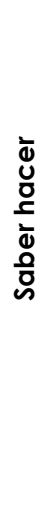 \\
\hline 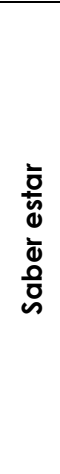 & $\begin{array}{l}\text { Competencia social } \\
\text { - Capacidad comunicativa } \\
\text { - Capacidad para comunicarse en una } \\
\text { segunda lengua } \\
\text { - Relaciones interpersonales } \\
\text { - Negociación } \\
\text {-Trabajo en equipo } \\
\text {-Liderazgo } \\
\text {-Resolución de problemas } \\
\text {-Empatía } \\
\text {-Autoridad } \\
\text {-Orientación a familias }\end{array}$ & $\begin{array}{l}\text { Competencia personal } \\
\text {-Autocontrol y estabilidad emocional } \\
\text {-Confianza y seguridad en sí mismo } \\
\text {-Resistencia ante las adversidades } \\
\text {-Iniciativa y espíritu emprendedor } \\
\text {-Capacidad de trabajo } \\
\text {-Influencia } \\
\text {-Visión y anticipación frente a la realidad } \\
\text {-Compromiso ético } \\
\text {-Toma de decisiones } \\
\text {-Flexibilidad, adaptación al cambio }\end{array}$ & 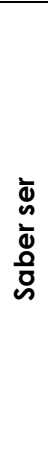 \\
\hline
\end{tabular}

La validez de contenido del instrumento se evidencia al desarrollar un proceso de validación con expertos en dos rondas, lo que permitió alcanzar consenso y estabilidad en las respuestas. 23 expertos fueron los que conformaron el panel, estando adscritos a distintas universidades, de diferentes disciplinas, todos ellos con conocimiento y experiencia en el ámbito educativo y, específicamente, en la formación de maestros. El 78\% son doctores, y entre ellos destacamos dos catedráticos de universidad, dos expertos en Filología, tres responsables del Prácticum en distintas universidades y tres directivos de centros educativos de Primaria de esta región. Todos ellos contaron con una pauta para evaluar, de 1 a 3 , la claridad y pertinencia de las variables e indicadores sobre las que tenían que pronunciarse, así como un espacio para proponer cambios o sugerencias.

Además, los valores de fiabilidad para el conjunto total de los ítems en cada una de las escalas es ( $a=.910$ para el grado de importancia y $a=.965$ para el nivel de logro), puntuaciones que confirman una alta consistencia interna por exceder el valor mínimo de 0,7 (DeVellis, 2003; Nunnally y Bernstein, 1995 y Pardo y Ruiz, 2010). 
En la Tabla 3 especificamos los valores de consistencia interna de cada una de las dimensiones, expresada por el coeficiente de alfa de Cronbach.

Tabla 3: Coeficiente de fiabilidad de las dimensiones del cuestionario

\begin{tabular}{lcl} 
& Grado de importancia & Nivel de logro \\
\hline Cognitiva & .770 & .832 \\
Metodológica & .774 & .891 \\
Social & .738 & .910 \\
Personal & .838 & .940 \\
\hline TOTAL & 0,910 & .965 \\
\hline
\end{tabular}

A continuación, se ha investigado la validez de constructo del cuestionario mediante un análisis de componentes principales. Los resultados advierten que el cuestionario no tenía una estructura unifactorial sino que evidencian la presencia de once factores para la escala del grado de importancia y, seis para la del nivel de logro. Estos explican respectivamente el $57 \%$ y $61,7 \%$ de la varianza. Este porcentaje se considera dentro de lo esperable y se ve influido por varios factores. De los cuales se destaca la dificultad de definir la competencia profesional del maestro por ser multidimensional y polisémica pues incluye necesariamente factores relacionados con conocimientos, actitudes y aptitudes. Así, los resultados coinciden con otros estudios en el sentido de encontrar numerosas dimensiones en el constructo (Fernández-Berrueco y Sánchez-Tarazaga, 2014; Perrenoud, 2004 y Tejada, 2009). En síntesis, los datos obtenidos permiten afirmar que el instrumento es adecuado

El estudio estadístico de los datos se ha basado en un análisis estadístico descriptivo mediante medidas de tendencia central y de dispersión de las variables (para conocer la valoración sobre la importancia y el nivel de logro de cada una de las competencias) e inferencial a través de la prueba † de Student, con el objeto de conocer si existen diferencias estadísticamente significativas entre lo deseable y lo logrado. Toda la información ha sido procesada a través del paquete estadístico SPSS v. 22.0. (2013).

\subsection{Procedimiento}

En cuanto a la aplicación del instrumento para la recogida de datos, se llevó a cabo durante los meses de febrero, marzo y abril del curso 2013 - 2014, periodo en que los estudiantes del Grado en Educación Primaria están realizando sus prácticas y se les asigna un futor en su centro de referencia. Destacar también que todos los cuestionarios recogidos 
son válidos sin encontrar valores perdidos en el global de la muestra $(n=131)$.

\section{RESULTADOS}

Antes de analizar los resultados obtenidos en cada una de las 4 dimensiones mencionadas, presentamos los resultados globales obtenidos de acuerdo al grado de importancia y nivel de logro de las competencias profesionales de nuestros estudiantes, tal como la perciben los tutores (Tabla 4). Se ha obtenido la puntuación de cada dimensión sintetizando las puntuaciones de cada ítem que la conforman.

Tabla 4: Estadísticos descriptivos de las dimensiones

\begin{tabular}{ccccccccccc}
\hline & \multicolumn{4}{c}{$\begin{array}{c}\text { Grado } \\
\text { importancia }\end{array}$} & \multicolumn{1}{c}{ Nivel de logro } \\
\hline & Media & D.T. & C.V. & Media & D.T & C.V. & $\begin{array}{c}\text { Diferencias } \\
\text { medias }\end{array}$ & $\begin{array}{c}\text { Correlación } \\
\text { Pearson }\end{array}$ \\
\hline Competencia didáctico- & & & & & & & & & \\
metodológica & 3.75 & .47 & 12.56 & 2.98 & .84 & 27.45 & $.77^{* *}$ & .082 \\
Competencia social & 3.72 & .49 & 13.1 & 2.84 & .88 & 29.43 & $.87^{* *}$ & $.152^{* *}$ \\
Competencia personal & 3.67 & .47 & 12.63 & 2.77 & .87 & 29.59 & $.89^{* *}$ & $.186^{* *}$ \\
Competencia cognitiva & 3.67 & .5 & 13.55 & 3.08 & .81 & 25.79 & $.599^{* *}$ & $.178^{* *}$ \\
\hline
\end{tabular}

Nota: $C V=$ coeficiente de variación. ${ }^{* *} p<.01$

Se observa que, respecto al grado de importancia, todas las dimensiones puntúan con medias que oscilan entre $\mu=3.67$ y 3.75 en la escala 1-4, que, junto a los datos de desviación típica y del coeficiente de variación, indica la homogeneidad en las respuestas. Destacan dos dimensiones como mejor puntuadas: la metodológica (saber hacer), es decir, utilizar los procedimientos metodológicos -de aprendizaje y evaluación-, adecuados a los alumnos, y saber transferir lo adquirido a nuevas situaciones y la competencia cognitiva (saber) que avala los conocimientos teóricos necesarios para saber fundamentar y articular nuestras decisiones y acciones pedagógicas. En cambio, queda ya en tercer lugar la competencia social (saber estar), que se refiere a aspectos comunicativos y actitudinales que permiten a la persona interactuar en el puesto de trabajo y desarrollarse profesionalmente con distintos equipos de trabajo. Y con una diferencia de 5 puntos, la competencia personal (saber ser), que incorpora características personales para realizar un trabajo de calidad con responsabilidad ética ante alumnos y familias. Resulta significativo que competencias claves para la gestión del aula y la interrelación con el contexto en el que trabajan queden situadas en el tercer y cuarto puesto. 
Con relación al nivel de logro las medias oscilan entre $\mu=2.77$ y 3.08 , resultando también de escaso valor estas diferencias. Además, los profesores tutores manifiestan que la competencia cognitiva $(\mu=3.08)$ es la más lograda, mientras que perciben como menos desarrollada la competencia personal $(\mu=2.77)$, que vuelve a situarse en el último lugar. Este dato es de gran interés, dado que la competencia menos valorada como importante $(\mu=3.67)$ se presenta como la más lograda, si bien, la puntuación no es elevada. De ahí se obtiene que si se compara los resultados entre la importancia y el logro, la menor diferencia de medias se da en la competencia cognitiva, por tanto existe una mayor adecuación entre lo deseable y lo logrado. El valor de la † de Student para muestras relacionadas revela que estas diferencias son estadísticamente significativas en las cuatro dimensiones.

En cuanto al análisis de correlación se ha encontrado una asociación lineal estadísticamente significativa, entre las puntuaciones del grado de importancia de las competencias social, personal y cognitiva y el nivel de logro de las mismas, cuyo valor de coeficiente de Pearson es bajo.

\subsection{Resultados descriptivos por dimensiones: importancia y logro para tutores}

Si se analizan cada una de estas dimensiones por separado, nos encontramos con los siguientes resultados.

\section{Competencia cognitiva}

En la dimensión competencia cognitiva, se aprecia el valor promedio más alto referido al dominio de la disciplina que enseña, identificación con el centro educativo y creatividad (Tabla 5). La menor concentración en torno a la media y, por tanto, el mayor nivel de disenso de entre estas tres, se produce en las dos últimas. Sin embargo, en la primera de ellas hay un alto grado de acuerdo y unanimidad puesto que los valores de desviación típica y coeficiente de variación son inferiores al resto. La mayor dispersión se da en la identificación con el centro educativo (D.T.=.95; CV=24.67).

Por otra parte, la competencia menos valorada es el conocimiento de la legislación vigente. A pesar de ello en torno al 50\% de los encuestados la han evaluado con la puntuación máxima (4), por lo que sí que es considerada importante, aunque en menor medida que el resto. 
Tabla 5: Estadísticos descriptivos de la competencia cognitiva.

\begin{tabular}{|c|c|c|c|c|c|c|c|c|}
\hline \multirow[t]{2}{*}{ Indicadores } & \multicolumn{3}{|c|}{ Grado de importancia } & \multicolumn{3}{|c|}{ Nivel de logro } & \multirow[b]{2}{*}{$\begin{array}{l}\text { Diferencia } \\
\text { medias }\end{array}$} & \multirow[b]{2}{*}{$\begin{array}{c}\text { Correlación } \\
\text { Pearson }\end{array}$} \\
\hline & Medias & D.T. & C.V. & Medias & D.T. & C.V. & & \\
\hline $\begin{array}{c}\text { Dominio de la } \\
\text { disciplina que enseña. }\end{array}$ & 3.89 & .32 & 8.22 & 3.28 & .79 & 25.79 & $.61^{* *}$ & .037 \\
\hline $\begin{array}{l}\text { Identificación con el } \\
\text { centro educativo. }\end{array}$ & 3.85 & .95 & 24.67 & 2.9 & .96 & 30.71 & $.95^{* *}$ & .165 \\
\hline Creatividad. & 3.82 & .82 & 21.46 & 3.08 & .85 & 27.54 & $.74^{* *}$ & .134 \\
\hline Conocimiento de la & & & & & & & & \\
\hline $\begin{array}{l}\text { Psicología del } \\
\text { Desarrollo. }\end{array}$ & 3.72 & .5 & 13.44 & 3.37 & .81 & 24.03 & $.35^{* *}$ & $.223^{* *}$ \\
\hline $\begin{array}{l}\text { Capacidad reflexiva, } \\
\text { autocrítica y crítica. }\end{array}$ & 3.66 & .53 & 14.48 & 3.05 & .8 & 26.22 & $.61^{* *}$ & $.278^{* *}$ \\
\hline $\begin{array}{l}\text { Capacidad de } \\
\text { análisis y síntesis. }\end{array}$ & 3.63 & .53 & 14.6 & 3.11 & .79 & 24.08 & $.52^{* *}$ & $.176^{*}$ \\
\hline $\begin{array}{l}\text { Conocimiento de los } \\
\text { distintos modelos de } \\
\text { instrucción. }\end{array}$ & 3.54 & .55 & 15.53 & 3.11 & .82 & 26.36 & $.43^{* *}$ & .081 \\
\hline $\begin{array}{l}\text { Conocimiento del } \\
\text { Centro educativo. }\end{array}$ & 3.52 & .56 & 15.9 & 2.8 & .86 & 25.4 & $.72^{* *}$ & .167 \\
\hline $\begin{array}{l}\text { Conocimiento sobre } \\
\text { la legislación } \\
\text { educativa vigente. }\end{array}$ & 3.4 & .67 & 19.7 & 3.05 & .84 & 33.1 & $.35^{* *}$ & $.332^{* *}$ \\
\hline
\end{tabular}

Las que reflejan medias más altas en el nivel de logro son el conocimiento de la psicología del desarrollo y el dominio de la disciplina, pero, a la vez, presentan mayor dispersión de los datos, lo que nos indica la existencia de un menor consenso. Las dos se refieren a conocimientos disciplinares, lo cual confirma que la formación recibida ha sido orientada a los conocimientos de naturaleza conceptual; es decir, el énfasis de la formación en la teoría. Este resultado permite situar estos hallazgos en consonancia con estudios que concluyen que estamos ante una competencia esencial (García y Martín, 2011; Marín, Latorre y Blanco 2010; Martín del Pozo, et al., 2013; Pesquero, et al., 2008). La valoración alta de esta competencia asociada al ámbito del saber sugiere un perfil profesional del maestro más próximo al modelo tradicional que al que se demanda en la actualidad (Ferrández y Sánchez, 2014), lo que nos lleva a incidir en una preparación más equilibrada entre las 4 dimensiones.

Al comparar las diferencias entre las medias de las dos escalas, se comprueba una mayor distancia entre ellas para identificación con el centro educativo y creatividad, sin duda elementos que no suelen trabajarse en el plan de estudios de la formación inicial. Además, respecto a la primera, el hecho de que la muestra esté conformada en 
su mayoría por tutores de centros privados y concertados podría explicar esta diferencia.

Por otro lado, aquellas competencias donde aparecen menos distancias en importancia y realidad tienen que ver con conocimiento de la psicología del desarrollo y conocimiento de la legislación vigente, siendo la primera muy valorada en el nivel de logro como ya se ha dicho, mientras que la segunda aparece como la menos valorada tanto en la escala importancia como en la de logro. El estadístico t de Student revela la existencia de diferencias significativas en los indicadores.

\section{Competencia didáctico-metodológica}

En cuanto a la competencia didáctico-metodológica, como se refleja en la Tabla 6, las medias más altas y las que tienen mayor homogeneidad en las respuestas, son aquellas relacionadas con el aula: orientación y acción tutorial y capacidad pedagógica; promoción de la convivencia; orientación académica y personal del alumnado. Esto expresa la importancia que otorgan los maestros a los recursos pedagógicos para diagnosticar, motivar, asesorar y orientar a cada alumno en su proceso personal, así como saber resolver problemas de disciplina y fomentar la convivencia en el aula y fuera de ella. Además, dentro de las competencias menos valoradas aparece dominio de las TIC y conocimiento de distintas metodologías lo cual parece un resultado contrario a las tendencias educativas del momento y a las exigencias planteadas en diferentes escenarios.

El análisis de correlación evidencia que el valor del coeficiente de Pearson es bajo se han encontrado relaciones estadísticamente significativa, entre importancia y logro en conocimiento de estrategias de aprendizaje, evaluación del proceso de enseñanza-aprendizaje, planificación y organización y dominio de las TIC.

Tabla 6: Estadísticos descriptivos de la competencia didáctico-metodológica

\begin{tabular}{ccccccccc}
\hline Indicadores & $\begin{array}{c}\text { Grado de } \\
\text { importancia }\end{array}$ & \multicolumn{7}{c}{ Nivel de logro } \\
\hline & Medias & D.T. & C.V. & Medias & D.T. & C.V. & $\begin{array}{c}\text { Diferencia } \\
\text { medias }\end{array}$ & $\begin{array}{c}\text { Correlaciones } \\
\text { Pearson }\end{array}$ \\
\hline $\begin{array}{c}\text { Orientación y Acción } \\
\text { Tutorial. }\end{array}$ & 3.89 & .85 & 21.85 & 2.99 & .91 & 30.43 & $.90^{* *}$ & .019 \\
\hline $\begin{array}{c}\text { Capacidad } \\
\text { pedagógica. }\end{array}$ & 3.88 & .32 & 8.24 & 3.17 & .83 & 27.45 & $.71^{* *}$ & -.009 \\
\hline $\begin{array}{c}\text { Promoción de la } \\
\text { Convivencia. }\end{array}$ & 3.85 & .36 & 9.35 & 2.77 & .9 & 32.49 & $1.08^{* *}$ & .080 \\
\hline $\begin{array}{c}\text { Orientación } \\
\text { académica y }\end{array}$ & 3.85 & .38 & 9.8 & 2.98 & .89 & 29.86 & $.87^{* *}$ & .083 \\
\hline
\end{tabular}




\begin{tabular}{|c|c|c|c|c|c|c|c|c|}
\hline $\begin{array}{l}\text { personal del } \\
\text { alumnado. }\end{array}$ & & & & & & & & \\
\hline $\begin{array}{l}\text { Atención a la } \\
\text { Diversidad. }\end{array}$ & 3.82 & .40 & 10.47 & 2.96 & .9 & 30.4 & $.86^{* *}$ & .085 \\
\hline $\begin{array}{l}\text { Conocimiento de } \\
\text { estrategias de } \\
\text { aprendizaje. }\end{array}$ & 3.73 & .47 & 12.56 & 2.96 & .86 & 29.05 & $.77^{* *}$ & $.255^{* *}$ \\
\hline $\begin{array}{l}\text { Evaluación del } \\
\text { proceso de } \\
\text { enseñanza- } \\
\text { aprendizaje. }\end{array}$ & 3.73 & .48 & 12.86 & 3.04 & .84 & 27.63 & $.69 * *$ & $.196^{*}$ \\
\hline $\begin{array}{l}\text { Planificación y } \\
\text { organización. }\end{array}$ & 3.7 & .49 & 13.24 & 3.09 & .84 & 27.18 & $.61^{* *}$ & $.307^{* *}$ \\
\hline $\begin{array}{l}\text { Conocimiento de } \\
\text { distintas } \\
\text { metodologías. }\end{array}$ & 3.69 & .51 & 13.82 & 3.02 & .81 & 26.82 & $.67^{* *}$ & .072 \\
\hline Dominio de las TIC. & 3.44 & .61 & 17.73 & 2.86 & .87 & 30.41 & $.58^{* *}$ & $.173^{*}$ \\
\hline
\end{tabular}

Nota: $C V=$ coeficiente de variación. ${ }^{*} p<0.05 ;{ }^{* *} p<0.01$

\section{Competencia social}

En el análisis de la competencia social, se aprecia que el promedio más alto y con menor desviación típica lo obtienen: orientación a familias, capacidad comunicativa y resolución de problemas, lo que responde a un mayor acuerdo entre los tutores participantes (Tabla 7).

Cabe subrayar que de las cuarenta competencias del cuestionario, la orientación a las familias es la que ha obtenido la media más alta y la dispersión más baja, ya que el $100 \%$ de los tutores la consideran muy importante; sin embargo, no obtiene unos valores de logro en sintonía con esta relevancia. Este alto reconocimiento pone de relieve un elemento fundamental que confirma que la implicación de las familias favorece los resultados escolares y se identifica como un indicador clave de calidad educativa, tal como se recoge en el último informe del Consejo Escolar del Estado (2014). En este sentido, se coincide en señalar la escasa preparación de los maestros y proponen la inclusión de estas competencias en su formación inicial, pues son ellos el puente de unión entre las familias y la escuela.

También tienen valores muy relevantes en la escala importancia la capacidad comunicativa y resolución de problemas, que tampoco aparece percibido en la misma medida en el nivel de logro. En contrapartida, llama la atención que algunas competencias relevantes para el maestro como liderazgo, comunicarse en una segunda lengua y capacidad de negociación, obtengan las medias más bajas en la visión del maestro tutor. 
Es de destacar que en estas competencias es donde se han encontrado mayor distancia entre lo importante y la realidad, a excepción de trabajo en equipo, punto que debe llevarnos a reflexionar sobre la formación que se está impartiendo en nuestras facultades y si está respondiendo a las necesidades de nuestra sociedad. Si los últimos informes e investigaciones coinciden en que gran parte de los problemas del aula se solventarían con la formación del profesorado en competencias sociales, deberíamos hacer un esfuerzo por incluirlas, de un modo u otro, en la formación inicial de nuestros maestros. En cuanto a trabajo en equipo y capacidad pedagógica, investigaciones precedentes también han puesto de relieve estos mismos hallazgos avalando la necesidad de ambas competencias en este entorno profesional (Agüera, Alfageme y Calderón, 2006; Martín del Pozo y De Juanas, 2009; Pesquero, et al., 2008; Valdemoro y Lucas, 2014).

Las relaciones estadísticamente significativas del análisis de la correlación se muestran en la tabla 7.

Tabla 7: Estadísticos descriptivos de la competencia social

\begin{tabular}{|c|c|c|c|c|c|c|c|c|}
\hline \multirow[t]{2}{*}{ Indicadores } & \multicolumn{3}{|c|}{$\begin{array}{c}\text { Grado de } \\
\text { importancia }\end{array}$} & \multicolumn{3}{|c|}{ Nivel de logro } & \multirow[b]{2}{*}{$\begin{array}{l}\text { Diferencias } \\
\text { medias }\end{array}$} & \multirow[b]{2}{*}{$\begin{array}{c}\text { Correlación } \\
\text { Pearson }\end{array}$} \\
\hline & $\begin{array}{c}\text { Media } \\
\mathrm{s}\end{array}$ & D.T. & C.V. & Medias & D.T. & C.V. & & \\
\hline $\begin{array}{l}\text { Orientar e implicar a } \\
\text { las familias. }\end{array}$ & 3.94 & .24 & 6.09 & 2.84 & .99 & 6.09 & $1.1^{* *}$ & .023 \\
\hline $\begin{array}{l}\text { Capacidad } \\
\text { comunicativa. }\end{array}$ & 3.89 & .38 & 9.76 & 2.99 & .89 & 9.76 &. $.9^{* *}$ & $.177^{*}$ \\
\hline $\begin{array}{l}\text { Resolución de } \\
\text { problemas. }\end{array}$ & 3.86 & .36 & 9.32 & 2.85 & .88 & 9.32 & $1.01^{* *}$ & .104 \\
\hline Trabajo en equipo. & 3.84 & .4 & 10.41 & 3.17 & .79 & 10.41 & $.67^{* *}$ & .084 \\
\hline Autoridad. & 3.82 & .44 & 11.5 & 2.83 & .97 & 11.5 & $.99 * *$ & .141 \\
\hline $\begin{array}{l}\text { Capacidad } \\
\text { investigadora }\end{array}$ & 3,76 & .44 & 11.70 & 3,09 & .86 & 27,83 & $.97 * *$ & $.257^{* *}$ \\
\hline Empatía. & 3.79 & .4 & 10.55 & 2.82 & .98 & 10.55 & $.85^{* *}$ & $.252^{* *}$ \\
\hline $\begin{array}{c}\text { Relaciones } \\
\text { interpersonales. }\end{array}$ & 3.69 & .53 & 14.36 & 2.84 & .89 & 14.36 & $.8^{* *}$ & $.281^{* *}$ \\
\hline Negociación. & 3.49 & .54 & 15.47 & 2.69 & .86 & 15.47 & $.78^{* *}$ & $.279 * *$ \\
\hline $\begin{array}{l}\text { Capacidad de } \\
\text { comunicarse en una } \\
\text { segunda lengua. }\end{array}$ & 3.48 & .63 & 18.1 & 2.7 & .9 & 18.1 & $.72^{* *}$ & $.329 * *$ \\
\hline Liderazgo. & 3.46 & .64 & 18.49 & 2.74 & .85 & 18.49 & & $.230 * *$ \\
\hline
\end{tabular}

\section{Competencia personal}

Por último, en el análisis de la competencia personal, las más valoradas en la escala importancia son capacidad de trabajo y compromiso ético con una baja oscilación en las respuestas (Tabla 8). 
Además, ambas son percibidas con un mayor nivel de logro por los tutores participantes. Respecto a las menos valoradas como importantes destacan visión y anticipación e influencia personal, que por otro lado son aquellas en las que se muestran también los valores inferiores de logro.

Tabla 8: Estadísticos descriptivos competencia personal

\begin{tabular}{|c|c|c|c|c|c|c|c|c|}
\hline \multirow[t]{2}{*}{ Indicadores } & \multicolumn{3}{|c|}{ Grado de importancia } & \multicolumn{5}{|c|}{ Nivel de logro } \\
\hline & $\begin{array}{c}\text { Media } \\
\mathrm{s}\end{array}$ & D.T. & C.V. & Medias & D.T. & C.v. & $\begin{array}{l}\text { Diferencia } \\
\text { medias }\end{array}$ & $\begin{array}{l}\text { Correlación } \\
\text { Pearson }\end{array}$ \\
\hline Capacidad de trabajo. & 3.88 & .32 & 8.24 & 3.02 & .87 & 28.8 & $.86^{* *}$ & .010 \\
\hline Compromiso ético. & 3.82 & .42 & 10.49 & 3.07 & .97 & 31.59 & $.75^{* *}$ & .019 \\
\hline Autocontrol y estabilidad & 3.77 & .42 & 11.14 & 2.62 & .97 & 37.02 & $1.15^{* *}$ & -.027 \\
\hline Confianza y seguridad & 3.76 & .42 & 11.17 & 2.79 & .99 & 35.48 & $.97^{* *}$ & .061 \\
\hline $\begin{array}{l}\text { Resistencia ante las } \\
\text { adversidades. }\end{array}$ & 3.73 & .46 & 12.33 & 2.63 & .84 & 31.93 & $1.1^{* *}$ & .075 \\
\hline $\begin{array}{l}\text { Flexibilidad, adaptación al } \\
\text { cambio. }\end{array}$ & 3.7 & .45 & 12.16 & 2.82 & .85 & 30.14 & $.88^{* *}$ & .154 \\
\hline Toma de decisiones. & 3.63 & .49 & 13.49 & 2.86 & .82 & 28.67 & $.77^{* *}$ & .139 \\
\hline $\begin{array}{l}\text { Iniciativa y espíritu } \\
\text { emprendedor. }\end{array}$ & 3.56 & .58 & 16.29 & 2.73 & .83 & 30.4 & $.83^{* *}$ & .123 \\
\hline Visión y anticipación & 3.49 & .54 & 15.47 & 2.59 & .85 & 32.81 & $.9 * *$ & .088 \\
\hline Influencia personal & 3.36 & .64 & 19.04 & 2.62 & .75 & 28.62 & $.74^{* *}$ & $.250 * *$ \\
\hline
\end{tabular}

Nota: $C V=$ coeficiente de variación. ${ }^{* *} p<.01$

Por otra parte, llama la atención que la competencia autocontrol y estabilidad percibida como la tercera más importante, aparece en el nivel de logro con uno de los valores más bajos junto a resistencia ante las adversidades ya que, en coherencia con el estudio de García (2010), para un trabajo docente hecho con profesionalidad y calidad es necesario el equilibrio emocional, el bienestar psicológico y el compromiso con la profesión. Quizá la razón sea que esta dimensión aglutina aspectos difíciles de trabajar desde la formación inicial del profesorado, pero que, no por ello, podemos dejar de lado. Urge incluir en el diseño de este título acciones formativas dirigidas al desarrollo y consolidación de estas competencias personales especificas del maestro, necesarias en un contexto donde la celeridad de los cambios y la diversidad son las notas características del entorno profesional en el que van a trabajar.

\section{DISCUSIÓN Y CONCLUSIONES}

Este análisis pone de manifiesto que la percepción de los tutores de prácticas recogen los resultados obtenidos en las investigaciones ya mencionadas, lo que corrobora la validez de este estudio, pero, a la vez, 
nos lleva a reflexionar sobre el diseño tan tradicional del Grado en Magisterio en Educación Primaria, en los que continuamos dando mayor relevancia a las dimensiones cognitiva y técnico-metodológica, que a la social y personal. En este punto destacamos una incoherencia entre las competencias que se destacan como imprescindibles para la gestión del aula y la atención de colectivos cada vez más diversificados, y los diseños de este título. Si contrastamos estos diseños, incluida la propuesta del Ministerio en su normativa para la formación de maestros (ORDEN $\mathrm{ECl} / 3857 / 2007)$, con la formación permanente que se ofrece a este colectivo, volvemos a detectar esta misma incoherencia. Este es un punto que debemos resaltar para ofrecer una formación inicial de los profesores de Primaria acorde con la realidad en la que van a trabajar.

Por otro lado, sorprenden algunas valoraciones que evidencian un menor grado de importancia en algunas competencias consideradas básicas para el ejercicio profesional. Por ejemplo, saber aplicar las tecnologías al ámbito educativo o el dominio de las TIC, que han sido destacadas con amplitud en la literatura al uso (Cabero y Marín, 2014; Martín, 2014; Perrenoud, 2004) no son, sin embargo, incluidas entre las competencias imprescindibles del maestro del siglo XXI, maestro que trabajará necesariamente en entornos digitales. Otras competencias, aunque sea paradójico, han sido percibidas como menos importantes son liderazgo, comunicarse en una segunda lengua y capacidad de negociación, situación que se confirma en otros trabajos (ANECA, 2004; Ferrández y Sánchez, 2014; Marín, Latorre y Blanco, 2010; Más Torrelló, 2012; Rosales, 2014). Ahora bien, esto denota que aún no hemos sabido incorporarlas en las aulas, ni demostrar a los docentes en ejercicio el valor de las mismas para el logro de una enseñanza de mayor calidad. Sin duda, volvemos a estar ante competencias que debemos atender en estos momentos tanto en la formación inicial como en la permanente.

En relación al contraste entre las dos escalas, éste ha permitido analizar las diferencias entre la deseabilidad y la realidad. Se subraya como hallazgo de especial interés la diferencia entre la valoración y el logro de la implicación y orientación a las familias, así como orientación y acción tutorial y promoción de la convivencia. A la luz de estos hallazgos, se hace necesario realizar cambios en la formación inicial de los maestros para que puedan orientar de modo profesional a las familias, estar preparados para el adecuado desempeño de la función orientadora y tutorial, así como favorecer desde la práctica docente la convivencia y el clima de aula y centro. Además, los tutores como maestros en activo son conscientes de las carencias formativas en resolución de problemas, resistencia ante las adversidades y estabilidad emocional, que aunque no son específicas del plan de estudios, se deberían abordar, de una u otra forma, a lo largo de la carrera, tal como 
ya hemos indicado. En definitiva, compartimos que "(...) la calidad se consigue, entre otras razones, a partir de un sistema educativo con profesores que estén bien formados y que cuenten con un conjunto de competencias profesionales que pongan en práctica de manera adecuada [...]" (Ferrández, y Sánchez, 2014, p. 15).

Se puede concluir que indagar sobre la formación recibida en la universidad y analizar las carencias formativas invita a reflexionar y a seguir investigando sobre las competencias profesionales y las claves formativas, con objeto de fundamentar su pertinencia y sugerir propuestas que mejoren el contenido de la formación que se ofrece en los planes de estudio del Grado en Educación Primaria, tanto en escenarios formales como no formales de la universidad. Y, en especial, en el periodo de prácticas como espacio formativo de indudable riqueza por su potencial transformador al proporcionar oportunidades para experienciar la enseñanza en acción (El Kadri y Roth, 2015).

Este estudio abre la puerta a nuevas líneas de investigación que permitan contrastar estos hallazgos con las percepciones de otros agentes educativos implicados en la formación inicial del profesorado, así como incorporar elementos cualitativos para profundizar en el discurso de los participantes, con especial atención a los maestros en formación como eje de cambio y mejora del sistema educativo.

\section{REFERENCIAS BIBLIOGRÁFICAS}

Agüera, E., Alfageme, M., y Calderón, M. (2006). Competencias de los futuros maestros: Entre la realidad y la deseabilidad. En Aplicaciones prácticas de la convergencia europea. Comunicación en I Jornadas Nacionales de Intercambio de Experiencias Piloto de Implantación de Metodologías ECTS. Universidad de Extremadura, Badajoz.

ANECA (2004). El libro Blanco del Título de Grado de Magisterio. Recuperado de http://www.aneca.es/modal.eval/converdocs titulos.html

Bunk, G.P (1994). La transmisión de las competencias en la formación y el perfeccionamiento profesionales de la RFA. Revista Europea de Formación Profesional, (1), 8-14.

Cabero, J., y Marín, V. (2014). Miradas sobre la formación del profesorado en TIC. Enl@ce Revista venezolana de Información, Tecnología y Conocimiento, $11(2), 11-24$.

Cano, E. (2005). Cómo mejorar las competencias de los docentes. Barcelona, España: Graó. 
Cano, E. (2008). La evaluación por competencias en la educación superior. Profesorado. Revista de currículum y formación del Profesorado, 12(3),1-16. Recuperado de http://www.ugr.es/ recfpro/rev123COL1.pdf.

Consejo Escolar del Estado (2014). La participación de las familias en la educación escolar. Recuperado de http://www.mecd.gob.es/.../2014/.../20141119.../EstudioParticipacionCEE_Digit

Delors, J. (1996). La educación encierra un tesoro. Informe a la UNESCO de la Comisión para la Educación para el siglo XXI. Madrid, España: Santillana.

DeVellis, R. (2003). Scale development: Theory and applications (2a. Ed.). Newbury Park, CA, Estados Unidos: Sage.

Echeverría, B. (2002). Gestión de la competencia de acción profesional. Revista de Investigación Educativa, (20), 7-43.

Echeverría, B. (2005). Competencias de acción de los profesionales de la orientación. Madrid, España: ESIC.

El Kadri, M.S., \& Roth, W.M. (2015). The teaching practicum as a locus of multi-leveled, school-based transformation. Teaching Education, $26(1), 17-37$

Ferrández-Berrueco, R., y Sánchez-Tarazaga, L. (2014). Competencias docentes en Secundaria. Análisis de perfiles de profesorado. RELIEVE, 20(1), 1-20.

Freire, M., Teijeiro M., y Blázquez, F. (2012). Investigación y estudio sobre competencias profesionales: el caso de la universidad de la Coruña. A Coruña, España: Universidad de A Coruña.

García Rodríguez, M., y Martín Palacio, M. (2011). El Prácticum como elemento de desarrollo de las competencias profesionales en estudiantes de magisterio. Revista de Orientación Educacional, (25), 45-58.

García, E. (2010). Competencias éticas del profesor y calidad de la educación. Revista Electrónica Interuniversitaria de Formación del Profesorado, (13), 29-41.

González, M., Pesquero, E., Sánchez, M., Fernández, P., De Juanas, Á., y Martín Del Pozo, R. (2010). Las competencias de los maestros de Primaria: percepción de los estudiantes en prácticas. Investigación en la escuela, (72), 71-83.

González Sanmamed, M., y Fuentes Abeledo, E. (2011). El Prácticum en el aprendizaje de la profesión docente. Revista de Educación, (354), 47-70.

Hernández, R., Fernández, C., y Baptista, P. (2010). Metodología de la Investigación ( $5^{\mathrm{a}}$ ed.) Madrid, España: McGraw-Hill.

Hernández Martín, A.H. (2014). La formación del profesorado para la integración de las TIC en el currículum: nuevos roles, competencias y espacios de formación. En A. García-Valcárcel. (coord.) 
Investigación y tecnologías de la información y comunicación al servicio de la innovación educativa (pp. 33-56). Salamanca, España: Ediciones Universidad de Salamanca.

Lasnier. F. (2000). Réussir la formation par compétences. Montreal, Canadá: Guérin.

Latorre, M.J., y Blanco, J. (2011). El Prácticum como espacio de aprendizaje profesional para docentes en formación. REDU: Revista de Docencia Universitaria, 9(2), 35-54.

Lawson, T., Çakmak, M., Gündüz, M., \& Busher, H. (2015). Research on teachıng practicum-a systematıc review. European Journal of Teacher Education, 38(3), 392-407.

Lévy-Leboyer, C. (2003). Gestión de las Competencias. Cómo analizarlas, cómo evaluarlas, cómo desarrollarlas. Barcelona, España: Gestión 2000.

Marcelo, C. (2011). Modelos innovadores en la supervisión del Prácticum. En M. Raposo, M.E. Martínez, P.C. Muñoz, A. Pérez, y J.C. Otero. Evaluación y supervisión del Prácticum: el compromiso con la calidad de las prácticas (pp.21-42). Santiago de Compostela, España: Andavira.

Marín, V., Latorre, M.J. y Blanco, F.J. (2010). Las competencias profesionales de la Titulación de Magisterio vistas desde la óptica del alumno receptor. Estudio del caso de Educación Primaria. Estudios Sobre Educación, (19), 219-235.

Martín del Pozo, R, y De Juanas, A. (2009). La formación inicial en competencias valoradas por los maestros en activo. Revista electrónica Interuniversitaria de Formación del profesorado, (12), 59-69.

Martín del Pozo, R, Fernández, P., González, M., y De Juanas, A. (2013). El dominio de los contenidos escolares: competencia profesional y formación inicial de maestros. Revista de Educación, (360), 363-387.

Mas Torelló, O. (2012). Las competencias del docente universitario: la percepción del alumno, de los expertos y del propio protagonista. Revista de Docencia Universitaria, (10), 299-318.

Medina Rivilla, A. (2009). Formación y desarrollo de competencias básicas. Madrid, España: Universitas.

Melgarejo, J., Pantoja, A., y Latorre, P.A. (2014). Análisis de la calidad del Prácticum en los estudios de Magisterio desde la perspectiva del alumnado. Aula de Encuentro, (16), 53 - 70.

Mendoza, M. y Covarrubias, C.G. (2014). Formación inicial de maestros desde un modelo por competencias: el caso de España. Estudios Hemisféricos y Polares, 5(1), 1-25.

Nunnally, J.C. y Bernstein, I. (1995). Teoría psicométrica (3a . ed.). México, D.F., México: McGraw-Hill.

Pardo, A. y Ruiz, M. A. (2005). Análisis de datos con SPSS 13 Base. Madrid, España: McGraw-Hill. 
Perrenoud, P. (2004). Diez nuevas competencias para enseñar. Invitación al viaje. Barcelona, España: Graó.

Perrenoud, P. (2010). La formación del profesorado: un compromiso entre visiones inconciliables de la coherencia. Revista Interuniversitaria de Formación del Profesorado, 68. Recuperado de http://www.aufop.com/.../uploaded_fi.../artículos/1279237044.pd f.

Perrenoud, P. (2004). Desarrollar la práctica reflexiva. En el oficio de enseñar: profesionalización y razón pedagógica. Barcelona, España: Graó.

Pesquero, E., Sánchez, M.E, González, M., Martín, R., Guardia, S., Cervelló, J., Fernández, P., Martínez, M., y Varela, P. (2008). Las competencias profesionales de los maestros de Primaria. Revista Española de Pedagogía, (241), 447-466.

Raposo, M., y Zabalza, M.A. (2011). La formación práctica de estudiantes universitarios: repensando el Prácticum. Revista de Educación, (354), 17- 20.

ORDEN ECI/3857/2007, de 27 de diciembre, por la que se establecen los requisitos para la verificación de los títulos universitarios oficiales que habiliten para el ejercicio de la profesión de Maestro en Educación Primaria (BOE, 29 de diciembre).

Rodicio, M.L. e Iglesias, M. (2011). La formación en competencias a través del Prácticum: un estudio piloto. Revista de Educación, (354), 99124.

Rodriguez, J. Álvarez, V., Gil, J., y Romero, S. (2011). Necesidades del profesorado de la Universidad de Sevilla respecto a la enseñanza que requiere el EEES. Cultura y Educación, (23), 323-340.

Rosales, C. (2014). ¿ Cómo será mi profesión? Tendencias Pedagógicas, (23), 29-44.

Ruiz Corbella, M., y Naval, C. (2012). La formación basada en competencias en la Universidad. Fortalezas y debilidades de un modelo. En F. Gil y D. Reyero. (coords.). Libro homenaje al profesor José Antonio Ibáñez-Martín (pp. 308-323). Madrid, España: Biblioteca Online.

Sarramona, J. (2007). Las competencias profesionales del profesorado de secundaria. ESE. Estudios sobre educación, (12), 31-40.

Tejada, J. (2009). Competencias docentes. Profesorado. Revista de Currículum y Formación de Profesorado, 13(2), 1-15. Recuperado de http://www.ugr.es/ recfpro/rev132COL2.pdf.

Tejada, J. (2012). La alternancia de contextos para la adquisición de competencias profesionales en escenarios complementarios de educación superior: marco y estrategia. Educación XX1, (15), 17-40.

Tejada, J. (2013). Profesionalización docente en la universidad: implicaciones desde la formación. Revista de Universidad y 
Sociedad del Conocimiento (RUSC), (10),

170-184.

doi: http://dx.doi.org/10.7238/rusc.v10i1.1471

Tejada, J., y Carvalho, M.L. (2013). El Prácticum en la formación inicial de maestros: percepciones de los tutores de universidad de educación infantil y primaria. En P.C. Muñoz Carril, M. Raposo-Rivas, M. González Sanmamed, M.E. Martínez-Figueira, M. ZabalzaCerdeiriña, y A. Pérez-Abellás. Un Prácticum para la formación integral de los estudiantes (pp. 1583-1597). Santiago de Compostela, España: Andavira.

Tejada, J., y Ruiz, C. (2013). Significación del prácticum en la adquisición de competencias profesionales que permiten la transferencia de conocimiento a ámbitos propios de la acción docente. Profesorado. Revista de curriculum y formación del profesorado. 17(3), 91-110. Recuperado de http://www.ugr.es/ recfpro/rev173ART5.pdf

Tobón, S., y Fernández Tobón, J.L. (2002). Modelo pedagógico basado en competencias. Medellín, Colombía: Funorie Universitas.

Valdemoros, M., y Lucas, B. (2014). Competencias que configuran el perfil del docente de primaria. Análisis de la opinión del alumnado de Grado en Educación Primaria. Aula Abierta, 42(1), 53-60.

Valle, J., y Manso, J. (2013). Competencias clave como tendencia de la política educativa supranacional de la Unión Europea. Revista de Educación, número extraordinario, 12-33.

Zabalza, M.A. (2003). Competencias docentes del profesorado universitario. Calidad y desarrollo profesional. Madrid, España: Narcea.

Zabalza, M.A. (2011). El Prácticum en la formación universitaria: estado de la cuestión. Revista de Educación, (354), 21- 46. 


\section{Sobre las autoras:}

\section{María Luisa Barceló Cerdá}

Licenciada en Ciencias de la Educación (Universidad de Valencia). Diploma de Estudios Avanzados (Universidad de Córdoba). Máster en Asesoramiento Educativo familiar y Máster en Neuropsicología y Educación (Centro Universitario Villanueva). Posee una amplia experiencia profesional como directivo en Centros Educativos. Su línea de investigación doctoral versa sobre las competencias del maestro: evaluación y formación en el marco del EEES.

\section{Marta Ruiz-Corbella}

Doctora en Ciencias de la Educación por la Universidad Complutense. Profesora Titular de 'Teoría de la Educación' en la Facultad de Educación de la UNED. Miembro del Grupo de Investigación Educación Superior Presencial y a Distancia (ESPYD). Entre sus líneas de investigación destaca la formación del profesorado, la educación superior, la educación moral y cívica, y la educación a distancia, sobre los que ha publicado diversos artículos y monografías.

\section{Para citar este artículo}

Barceló Cerdá, M. L. \& Ruíz-Corbella, M. (2015). Las competencias profesionales del maestro de primaria desde la perspectiva del tutor del centro de prácticas. Revista Fuentes, 17 (Diciembre), 17-39. [Fecha de consulta: $\mathrm{dd} / \mathrm{mm} / \mathrm{aa}]$.

doi: doi: http://dx.doi.org/10.12795/revistafuentes.2015.117.01. 